\title{
Study on The Expression and Correlation of Alpha- Enolase (Eno1) in The Serum of Patients With Hepatocellular Carcinoma
}

Xia Luo

Department of Infectious Disease, General Hospital of Ningxia Medical University

Lina Ma

Department of Infectious Disease, General Hospital of Ningxia Medical University

Shuaiwei Liu

Department of Infectious Disease, General Hospital of Ningxia Medical University

Long Hai

Department of Infectious Disease, General Hospital of Ningxia Medical University

Xiangchun Ding ( $\nabla$ 13619511768@163.com)

Department of Infectious Disease, General Hospital of Ningxia Medical University

\section{Research Article}

Keywords: alpha-enolase, hepatocellular carcinoma, serum tumor marker, AFP

Posted Date: March 15th, 2021

DOl: https://doi.org/10.21203/rs.3.rs-279041/v1

License: (c) (i) This work is licensed under a Creative Commons Attribution 4.0 International License. Read Full License 


\section{Abstract}

Background: Serum tumor markers for the early detection of hepatocellular carcinoma is limited. This study analyzed the distribution and differences of ENO1 in the serum of healthy people, patients with chronic hepatitis B, cirrhosis and hepatocellular carcinoma. Furthermore, the distribution and correlation of ENO1 in the clinical data of patients with hepatocellular carcinoma were explored and its clinical diagnostic effect as well as combined diagnostic effect with AFP were compared. In addition, the effect of ENO1 on the survival of patients with hepatocellular carcinoma were also evaluated. The study will provide data basis for the application of ENO1 in the diagnosis of hepatocellular carcinoma, and provide new ideas for the study of molecular markers and therapeutic targets for hepatocellular carcinoma.

Methods: In accordance with the inclusion and exclusion criteria, Patients diagnosed at the Infectious Diseases Department of the General Hospital of Ningxia Medical University and the healthy check-up population at the medical check-up centre from May 2012 to March 2017 were screened for inclusion in the study. T The final study subjects were 181, 28 patients with chronic hepatitis B (CHB), 31 patients with liver cirrhosis (LC), 104 patients with liver cancer (HCC) and 18 healthy people (NC). Clinical data and sera were collected from all study subjects, tissue specimens were collected from some patients. The levels of serum ENO1 and AFP were measured by ELISA. QRT-PCR and Western Blot t were used to detect the relative expression of ENO1 mRNA and protein in liver cancer tissues and paracancerous tissues. Statistical analysis was performed by t-test, bivariate Spearman correlation test, curve regression analysis, ROC curve analysis and survival curve analysis.

Results: The relative expression of ENO1 mRNA and protein was significantly upregulated in hepatocellular carcinoma tissues relative to paraneoplastic tissues $(p<0.05)$, ENO1 levels in the serum of HCC patients were sequentially higher than those of CHB patients, LC patients and healthy subjects, with significant differences $(p<0.05)$. The differences in the distribution of serum ENO1 levels in the clinical data subgroups alpha fetoprotein (AFP) status, TNM stage (II vs III, III vs IV) were not statistically significant $(p>0.05)$, there were statistically significant differences $(p<0.05)$ in the distribution of gender, tumor size, whether the tumor was metastatic or not, TNM stage (I vs II) and Edmondson's grading in the clinical information subgroup. The difference in the distribution among clinical information subgroups of whether the tumor had recurred was highly significant $(p<0.01)$. Serum ENO1 levels showed a significant positive correlation with AFP levels (Spearman's correlation coefficient 0.312, $p=0.001^{\star *}$ ), the diagnostic sensitivity and specificity of ENO1 in this group of HCC patients were 0.673 and 0.831 (AUC=0.782, cutoff $=75.96, p=0.0001$ ), respectively, the sensitivity of ENO1 in conjunction with AFP in the diagnosis of $\mathrm{HCC}$ in this group was 0.740 and the specificity was 0.723 (AUC=0.835, $p=0.0001)$, the AUCs of ENO1 in the diagnosis of HCC tumor size, whether the tumor was metastatic, TNM I stage, Edmondson classification and whether the tumor recurred in this group were $0.663\left(0.546-0.781, P=0.012^{*}\right), 0.681$ (0.574-0.787, $\left.\mathrm{P}=0.002^{\star *}\right), 0.553(0.421-0.686, \mathrm{P}=0.386), 0.710(0.602-0.819, \mathrm{P}=0.001 * *)$, and 0.685 $\left(0.580-0.789, P=0.001^{\star *}\right)$. 
Conclusion: Serum ENO1 levels were significantly correlated with the occurrence, progression and metastasis of hepatocellular carcinoma, staging and postoperative survival of patients. ENO1 may be a candidate diagnostic marker for early diagnosis and evaluation of hepatocellular carcinoma progression, and can be used in combination with AFP as an auxiliary diagnostic function.

\section{Background}

Primary liver cancer ranks sixth in tumor incidence and is the second leading cause of death due to cancer, reports show that 905,677 cases of liver cancer were diagnosed and 830,180 deaths were reported worldwide in $202{ }^{[1]}$. Hepatocellular carcinoma (HCC) accounts for $90 \%$ of primary liver cancers. Hepatitis B virus (HBV) infection, hepatitis C virus (HCV) infection, liver cirrhosis, alcohol abuse, diabetes or obesity-related metabolic syndrome can all cause $\mathrm{HCC}^{[2-3]}$. According to statistics, the 5-year survival rate of Chinese liver cancer patients from 2003-2015 was only $12.5 \%{ }^{[4]}$. Currently, surgery remains the main treatment for hepatocellular carcinoma, but more patients are initially diagnosed at an intermediate to advanced stage and are unable to undergo effective treatment. Therefore, early detection and treatment can effectively improve the survival rate of hepatocellular carcinoma. Serum tumor markers are one of the early diagnostic modalities for hepatocellular carcinoma and can complement the pathological diagnosis of hepatocellular carcinoma, the combination of multiple serum markers can enable early detection of hepatocellular carcinoma tumor lesions. The existing serum markers for hepatocellular carcinoma (HCC) are mainly alpha fetoprotein (AFP). However, reports show that the diagnostic sensitivity of AFP is about $39-64 \%$, which to some extent limits the clinical utility of AFP [5]. Therefore, screening for markers or therapeutic targets for the early diagnosis of hepatocellular carcinoma is of great importance for the prevention and treatment of hepatocellular carcinoma.

A-enolase (a-enolase, ENO1) is one of the key enzymes of the pentose phosphate pathway and plays an important role in the process of cellular energy metabolism ${ }^{[6-7]}$. The function of ENO1 is distinguished by its location in the cell. If A-enolase is located in the cytoplasm, it acts as a rate-limiting enzyme of the glycolytic pathway, influencing the metabolism of tumor cells and thus regulating tumourigenesis and development ${ }^{[8]}$. If A-enolase is located on the cell surface, it acts as a fibrinogen receptor and promotes tumor cell invasion by activating fibrinolytic enzymes and accelerating the degradation of ECM ${ }^{[9]}$. If Aenolase is located in the nucleus, its encoded protein can influence tumourigenesis and development through transcriptional repression ${ }^{[10]}$. Studies have shown that ENO1 is aberrantly highly expressed in a variety of tumor cells, and that its high expression promotes proliferation, invasion and suppresses apoptosis ${ }^{[11]}$. In many tumors, ENO1 or anti-ENO1 antibodies have the potential to function as tumor markers. For example, Pan et al. identified ENO1 as a lung cancer-related protein marker for clinical application through semi-quantitative proteomic analysis ${ }^{[12]}$. ENO1 is highly expressed in ovarian cancer and can be used as a diagnostic marker and therapeutic target for ovarian cancer ${ }^{[13]}$. ENO1 has been associated with breast cancer, and a study by Mittal L et al. demonstrated that the expression of ENO1 was significantly reduced in triple-negative breast cancer treated with electrochemical therapy, accompanied by activation of related signaling pathways and ultimately apoptosis, suggesting that 
ENO1 may be a possible target for anticancer therapy in breast cancer ${ }^{[14]}$. A recent study, through bioinformatic analysis of the distribution and expression of ENO1 in hepatocellular carcinoma expression profile microarrays in public databases such as TCGA, found that ENO1 expression was significantly upregulated in hepatocellular carcinoma tissues and cells relative to normal liver tissues or hepatocellular carcinoma paracancerous tissues, and its expression was significantly correlated with the survival time

of patients ${ }^{[15-16]}$. Unfortunately, there are fewer reports on whether the detection rate of HCC can be improved by detecting the concentration of ENO1 in blood, and extensive in-depth studies are needed. Therefore, studying the distribution and correlation of serum ENO1 in clinical indicators of patients with hepatocellular carcinoma is of great significance for the early diagnosis and prevention of HCC.

This experiment intends to collect clinical data and sera from healthy people, patients with chronic hepatitis B, cirrhosis and hepatocellular carcinoma. The expression levels of ENO1 and AFP in their sera will be measured., to analyze the distribution and differences of ENO1 in the sera of healthy population, chronic hepatitis B, cirrhosis and hepatocellular carcinoma patients, to explore its distribution and correlation in the clinical data of hepatocellular carcinoma patients, compare its clinical diagnostic effect with AFP and combined diagnostic effect, and evaluate the effect of ENO1 on the survival of hepatocellular carcinoma patients. The study will provide theoretical and data basis for the application of ENO1 in the diagnosis and healing evaluation of hepatocellular carcinoma, and provide new ideas for the research of molecular markers and therapeutic targets of hepatocellular carcinoma.

\section{Methods}

\section{Study subjects and grouping}

The study subjects were from May 2012 to March 2017 at the Department of Infectious Diseases, General Hospital of Ningxia Medical University. Based on the inclusion and exclusion criteria, 181 cases were finally identified as eligible for enrollment in the study, 28 patients with chronic hepatitis B (CHB), 31 patients with liver cirrhosis (LC), 104 patients with liver cancer (HCC) and 18 cases in the healthy population (NC). All subjects signed an informed consent form prior to the survey sampling. The study protocol and all amendments were approved by the institutional review board or ethics committee of participating site and were conducted per the principles expressed in the Declaration of Helsinki. All patients provided written informed consent to participate.

\subsection{Criteria for diagnosis, inclusion and exclusion}

\subsubsection{Diagnostic conditions}

Chronic hepatitis B, cirrhosis and liver cancer were diagnosed according to the Asian Pacific Association for the Study of the Liver (APASL), the European Association for the Study of the Liver (EASL) and the American Association for the Study of Liver Diseases standards ${ }^{[17]}$. Liver function status was graded according to the Child Class score and the MELD (Model for end-stage liver disease, MELD) ${ }^{[18]}$. Patients 
with hepatocellular carcinoma were staged according to the American Joint Committee on Cancer 6th edition TNM staging criteria ${ }^{[19]}[$

\subsubsection{Criteria for inclusion and exclusion}

Criteria for patient inclusion: Patients who meet the diagnostic criteria of 1.1.1 and have a confirmed diagnosis of chronic hepatitis B, cirrhosis or hepatocellular carcinoma. Exclusion criteria for liver cancer are: $₫$ Liver cancer not due to hepatitis $B$ infection; खlmproper clinical information; $\bigotimes$ Patients with recent serious infections, surgery, trauma and other diseases. Exclusion criteria for chronic hepatitis $B$ are: $\triangle$ Patients with viral hepatitis not caused by hepatitis B virus; $\triangle$ Patients who have progressed to the cirrhotic stage of the liver.

QPatients with recent serious infections, surgery, trauma, etc.

\section{Research Methodology}

\subsection{Collection of general data}

Basic information was collected on all study subjects. This includes: Age, Gender, diagnosis, previous medical history (history of tumor, history of surgery). Anthropometric height measurement: After fasting overnight, study subjects will have their height measured on a meter on the day of the blood draw (refer to the Chinese Adult Body Mass Measurement Standard). The subject should be barefoot and stand in a standing position on the meter with both eyes level. Results of alanine transaminase (ALT) and aspartate transaminase (AST) tests were collected from all patients with liver disease. Clinical characteristics of 104 patients with liver cancer were collected: including tumor number, tumor size, Child Class score results, MELD score results and TNM stage results.

\subsection{ELISA Assay}

The study population of $163+8$ people ate regularly for 3 days prior to blood sampling, fasting for 8 hours on the day before the blood draw, $5 \mathrm{ml}$ of fasting venous blood was drawn on the following morning, within $2 \mathrm{~h}$ of the blood being drawn, the blood was taken to the laboratory, the serum is separated by centrifugation at $1000 \mathrm{rpm}$ for $5 \mathrm{~min}$ at room temperature. Using the ENO1 (alpha-enolase, alphaenolaSe) ELISA kit (abcam, UK) and AFP (alpha-fetoprotein) ELISA kits (Invitrogen, USA), serum ENO1 levels were measured by a multifunctional chemiluminescence instrument (Promega, USA) for each group of samples. The remaining blood specimens were centrifuged to obtain plasma and stored at -80 ${ }^{\circ} \mathrm{C}$. ELISA assay steps. $\mathbb{Q}$

(1) Remove the ELISA plate and allow to stand for 20 minutes at room temperature before equilibrating the plate components.

(2) Dilute the standards in a 2-fold gradient using sample diluent and add $50 \mathrm{uL}$ of diluted sample to each well using sample diluent. Take $10 \mathrm{uL}$ of serum sample and mix with $40 \mathrm{uL}$ of diluent and add to the 
assay wells.

(3) Seal the 96-well ELISA culture plate with air-seal plate film, shake and mix, centrifuge the plate transiently and incubate for $60 \mathrm{~min}$ in a $37^{\circ} \mathrm{C}$ thermostatic incubator for the reaction.

(4) Remove the plate, discard the liquid from the plate, pat the plate dry on stream filter paper, add $100 \mathrm{uL}$ of wash buffer per well and leave for $1 \mathrm{~min}$ at room temperature, discard the wash buffer and drain the plate again, repeat 5 times.

(5) Add $50 \mathrm{uL}$ each of substrate $A$ and substrate $B$ to the wells and incubate for 15 min at $37^{\circ} \mathrm{C}$ in an incubator protected from light, add $50 \mathrm{uL}$ of termination solution to terminate the reaction, read the OD value of the wells at $450 \mathrm{~nm}$ using a fluorescent enzyme marker, set up 3 replicate wells for each sample.

\subsection{RNA preparation and reverse transcription}

Lysis and extraction of total RNA from tissues using TRIzol ® Reagent (Invitrogen『USA) 『verify RNA quality by electrophoresis of $0.3 \mu \mathrm{g}$ RNA(1.3\% agarose gel electrophoresis, 1× TAE buffer, $120 \mathrm{~V}, 20 \mathrm{~min})$. The RNA was reverse transcribed into cDNA using the PrimeScript ${ }^{\circledR}$ RT reagent kit(Thermo®USA), the reverse transcribed RNA template was 500ng, The reverse transcription conditions are: 5 minutes at $25^{\circ} \mathrm{C}$, $42^{\circ} \mathrm{C}$ for 60 minutes and $70^{\circ} \mathrm{C}$ for 5 minutes, the remaining RNA and cDNA were stored at $-80^{\circ} \mathrm{C}$.

\subsection{Relative expression of EN01 gene mRNA in hepatocellular carcinoma tissues by QRT-PCR}

The cDNA was obtained from each group, the relative expression of EN01 gene was measured by QRTPCR using TransStart Tip Green qPCR SuperMix (Quan-Style Gold Biology, Beijing, China) on a LightCycler 480II quantification platform (Roche, USA). $\beta$-actin was used as the internal reference gene and the details of primer sequences used in the experiment were showed in Table1. The amount of cDNA template added within the reaction was $1 \mu \mathrm{L}$, Reaction conditions were $94^{\circ} \mathrm{C}$ for 30 seconds; $94^{\circ} \mathrm{C}$ reaction for $5 \mathrm{sec}, 60^{\circ} \mathrm{C}$ for $30 \mathrm{sec}, 72^{\circ} \mathrm{C}$ for $20 \mathrm{sec}, 40$ cycles of reaction; $65^{\circ} \mathrm{C}$ for 5 seconds. The Relative expression was calculated by the $2^{-\Delta \Delta C t}$ method.

\section{Table 1. Primer sequences used for QRT-PCR}

\begin{tabular}{|c|c|c|c|}
\hline Name of gene & & Primer Sequence $\left(5^{\prime}-3^{\prime}\right)$ & Size of outputs \\
\hline \multirow[t]{2}{*}{ EN01 } & Forward & CCTGTACCGCCACATCG & \multirow[t]{2}{*}{183 bp } \\
\hline & Reverse & TGGTAAACCTCTGCTCCAAT & \\
\hline \multirow[t]{2}{*}{$\beta$-actin } & Forward & CCACGGCTGCTTCCAGCTCC & \multirow[t]{2}{*}{131 bp } \\
\hline & Reverse & GGACTCCATGCCCAGGAAGGAA & \\
\hline
\end{tabular}

2.5 Western Blot detection of the relative expression of ENO1 protein in hepatocellular carcinoma tissues 
Total protein was extracted from liver cancer tissue using a Total Protein Extraction Kit (KGI, Nanjing, China). Grinding of tissue specimens to powder using liquid nitrogen in a mortar】add $400 \mu \mathrm{L}$ of lysate, grind again to a powder, After the lysate powder has thawed to a liquid, the liquid is collected, centrifuge in a tube for 10 minutes under the condition of $4^{\circ} \mathrm{C}$ and $12,000 \mathrm{rpm}$, The supernatant is the protein extract. The concentration of the protein extract was determined using a BCA protein quantification kit (Nanjing KGI, China). $20 \mu \mathrm{g}$ of protein specimens were taken, the proteins were separated in $10 \%$ SDSPAGE electrophoresis. The proteins were transferred onto PVDF membranes (Millipore Corp, USA) using a semi-dry transfer machine (Biorad Trans-Blot SD, BIO RAD, USA). A Sealing fluid was made by dissolving $50 \mathrm{~g}$ of skimmed milk powder in $100 \mathrm{ml}$ of $1 \mathrm{x}$ TBST, The incubator was closed for $3 \mathrm{~h}$, then the sealing fluid was discarded $\triangle$ Wash 3 times with $1 \times$ TBST and add ENO1 primary antibody (monoclonal antibody, 1:1000 dilution, abcam, UK) and $\beta$-actin primary antibody (monoclonal antibody, 1:1000 dilution, abcam, UK) to the incubation cassette. खThe incubation cassette was placed on a plate shaker at $50 \mathrm{rpm} 4^{\circ} \mathrm{C}$ at room temperature and incubated overnight, 1 x TBST washed 5 times for 5 min each $₫ A f t e r$ washing, IgG secondary antibody (1:5000 dilution, Proteintech, USA) was added to the incubation cassette区lncubation cassettes were placed on a plate shaker at $50 \mathrm{rpm}$ for $2 \mathrm{~h}$ and $1 \times$ TBST washed 5 times. 1:1 mixture of liquid $A$ and liquid $B$ of the ECL(WesternBright ECL囚advansta『USA)was prepared as the assay solution, The bands were observed after exposure of the PVDF membrane using a multicolor fluorescent gel formation system(ChemiDoc MP Imaging System®Bio-Rad, USA). The protein bands were quantified using Image $\mathrm{J}$ software.

\subsection{Statistical analysis}

Data were collated and analyzed by using SPSS 23.0 (IBM, USA) software, All measurements are presented as mean \pm standard deviation $(x \pm s)$, Comparative analysis of the significance of continuous measures and subgroup data using the T-test and one-way ANOVA methods; Analysis of the correlation between serum ENO1 levels and AFP levels using the bivariate Spearman correlation test; Analysis of the correlation between ENO1 levels and hepatitis B virus using curve regression; Analysis of the sensitivity and specificity of serum ENO1 in the diagnosis of hepatocellular carcinoma using the ROC curve (receiver operating characteristic curve); The survival curve was used to evaluate the relationship between serum ENO1 and survival of patients with hepatocellular carcinoma. All significant results were considered statistically significant at $p<0.05$ and highly significant at $p<0.01$.

\section{Results}

\section{Distribution of ENO1 in different liver diseases}

The expression levels of serum ENO1 were measured by ELISA and the clinically relevant test results were collected from the investigated subjects, as shown in Table 2. The results showed that the expression level of ENO1 in the serum of the healthy population was low, approximately $19.39 \pm 10.81 \mathrm{mg} / \mathrm{L}$. Serum ENO1 expression levels were significantly higher in the CHB group (80.70 \pm 90.79$)$, LC group (37.51 \pm 27.94$)$ and HCC group (154.06 \pm 166.74$)$ than in the NC group of the healthy population $(p<0.01)$, among them, 
the expression level of ENO1 was significantly higher in the HCC group than in the LC and CHB groups $(p<0.01)$. The results suggest that the development of liver diseases such as chronic hepatitis $B$, cirrhosis and hepatocellular carcinoma will lead to a significant increase in serum ENO1 expression levels, with the highest differential upregulation in hepatocellular carcinoma, and ENO1 has the potential to become a serum marker for early hepatocellular carcinoma.

\section{Table 2. General situation of the study subjects}

\begin{tabular}{|c|c|c|c|c|}
\hline Characteristics & $N C(n=18)$ & $\mathrm{CHB}(n=28)$ & $\operatorname{LC}(n=31)$ & $\operatorname{HCC}(n=104)$ \\
\hline Age (years) mean \pm SD & NA & $42.26 \pm 11.92$ & $51.10 \pm 8.86$ & $57.45 \pm 9.51$ \\
\hline Gender (male:female) & $13: 5$ & $20: 8$ & $26: 5$ & $88: 16$ \\
\hline $\begin{array}{l}\text { HBV DNA(log IU/mL), mean } \pm \\
\text { SD }\end{array}$ & NA & $4.96 \pm 1.90$ & $4.15 \pm 2.29$ & $3.03 \pm 1.50$ \\
\hline ALT (IU/L), mean \pm SD & NA & $268.36 \pm 440.01$ & $142.45 \pm 251.29$ & $72.22 \pm 114.65$ \\
\hline $\mathrm{AFP}(\mathrm{ng} / \mathrm{mL})$, mean $\pm \mathrm{SD}$ & NA & $43.66 \pm 80.36$ & $66.94 \pm 165.71$ & $501.36 \pm 533.71$ \\
\hline \multicolumn{5}{|l|}{ AFP Status $₫ \mathrm{n} \rrbracket$} \\
\hline AFP Negative & \multirow[t]{2}{*}{ NA } & \multirow[t]{2}{*}{ NA } & 14 & 21 \\
\hline AFP Positive & & & 17 & 83 \\
\hline $\mathrm{ENO1}(\mathrm{mg} / \mathrm{L})$, mean $\pm \mathrm{SD}$ & $19.39 \pm 10.81$ & $80.70 \pm 90.79$ & $37.51 \pm 27.94$ & $154.06 \pm 166.74$ \\
\hline Child Class $(\mathrm{A} / \mathrm{B} / \mathrm{C} /)$ or $\mathrm{NA} \%$ & NA & NA & $15: 5: 11$ & $36: 30: 38$ \\
\hline MELD score, mean \pm SD & NA & NA & $9.98 \pm 7.60$ & $7.98 \pm 5.65$ \\
\hline \multicolumn{5}{|l|}{ TNM Grading (n) } \\
\hline $\mid-\|$ & \multirow[t]{2}{*}{ NA } & \multirow[t]{2}{*}{ NA } & \multirow[t]{2}{*}{ NA } & 47 \\
\hline III-IV & & & & 57 \\
\hline \multicolumn{5}{|l|}{ Metastatic or not (n) } \\
\hline Yes & \multirow[t]{2}{*}{ NA } & \multirow[t]{2}{*}{ NA } & \multirow[t]{2}{*}{ NA } & 64 \\
\hline No & & & & 40 \\
\hline \multicolumn{5}{|l|}{ Recurrence or not (n) } \\
\hline Yes & NA & NA & NA & 32 \\
\hline No & & & & 72 \\
\hline
\end{tabular}

Note: NC- Negative control $₫$ CHB-Chronic Hepatitis B; LC- liver cirrhosis $₫$ HCC- Hepatocellular Carcinoma $₫$ HBV- hepatitis $B$ virus $₫ A L T$ - Alanine transaminase $\triangle A F P$ - alpha-fetoprotein囚ENO1-a-enolase $\triangle M E L D-M o d e l$ 
for end-stage liver disease $₫ \mathrm{TNM}$ - Tumor Node Metastasis; NA- Not applicable.

\section{Differential expression of ENO1 in healthy people/non-hepatocellular carcinoma liver disease patients/hepatocellular carcinoma patients and in hepatocellular carcinoma/paraneoplastic tissues}

The expression of ENO1 gene mRNA and protein levels in hepatocellular carcinoma and paraneoplastic tissues were detected by qRT-PCR and Western blot methods. The results showed that the expression level of serum ENO1 was significantly higher in the serum of patients with liver cancer than that of patients with non-hepatocellular carcinoma and the healthy population $(p=0.001)$ (Figure 1-A). The mRNA expression of ENO1 gene in paraneoplastic tissues was normalized to control base 1 and $\beta$-actin was used as an internal reference gene囚The mRNA expression level of ENO1 was significantly upregulated in hepatocellular carcinoma tissues relative to the paraneoplastic tissues $(p=0.001)$, with a difference of approximately 3.5-4.5-fold (Figure 1-B). Randomly selected cancer/paraneoplastic tissues from two patient, protein expression showed consistent expression results with ENO1 mRNA, i.e. the relative expression of ENO1 protein was significantly higher in liver cancer tissues than in paraneoplastic tissues $(p=0.003)$ (Figure 1-C). The correlation results further suggested that the up-regulation of ENO1 expression might be associated with the development of hepatocellular carcinoma.

1. Differences in expression of serum ENO1 levels in non-hepatocellular carcinoma patients (CHB patients, LB patients) and hepatocellular carcinoma patients, $p<0.05$. B. Relative expression of mRNA levels of ENO1 in hepatocellular carcinoma and paraneoplastic tissues, $p<0.05 ; C$. Relative expression of protein levels of ENO1 in hepatocellular carcinoma and paraneoplastic tissues, $p<0.05$. CHB- Chronic hepatitis B, LC- Liver cirrhosis, HCC- Hepatocellular carcinoma, NC- healthy population, PC- Para-carcinoma.

\section{Correlation of hepatitis B virus with serum ENO1 levels and its distribution in $\mathbf{3}$ groups of liver} diseases

The correlation between the distribution of serum ENO1 levels and hepatitis B virus levels in liver diseases in the CHB group, LC group and HCC group was investigated by curvilinear regression analysis. The results showed that serum ENO1 levels were positively correlated with hepatitis B virus DNA levels in the $\mathrm{CHB}, \mathrm{LC}$ and HCC groups, with the correlation between serum ENO1 levels and hepatitis B virus DNA levels in the CHB group being highly significant $(p=0.006)$, while the correlation was not statistically significant in the LC and HCC groups ( $p>0.05$ ) (Figure 2 ). The correlation results suggest that the elevated ENO1 levels may be associated with hepatitis B virus infection.

\section{Distribution of serum ENO1 levels in different clinical phenotypes of hepatocellular carcinoma}

Clinical data of 104 patients with hepatocellular carcinoma were grouped into age, type, AFP status, tumor size, whether the tumor was metastatic, whether the tumor was recurrent, TNM stage of the tumor, and Edmondson grading of the tumor囚The differences in the distribution of serum ENO1 levels among these clinical profiles of hepatocellular carcinoma were investigated by independent sample T-test test. The results showed that there was no statistically significant difference in the distribution of serum ENO1 
levels in AFP status and TNM stage (II vs III, III vs IV) in the clinical data groups ( $p>0.05)$, There were statistically significant differences $(p<0.05)$ in the distribution of gender, tumour size, whether the tumour was metastatic or not, TNM stage (I vs II) and Edmondson's classification in the clinical data group, the difference in the distribution of clinical data grouped by tumour recurrence was highly significant $(p<0.01)$. The results further suggest that serum ENO1 can be used as a marker for the diagnosis of earlystage hepatocellular carcinoma and the diagnosis of recurrence of hepatocellular carcinoma. In addition, the high expression of ENO1 in hepatocellular carcinoma may be associated with the process of hepatocellular carcinoma development, metastasis and recurrence, and the related mechanism is unknown. (Table 3)

Table 3. Distribution of serum ENO1 levels in the clinical phenotype of HCC 


\begin{tabular}{|c|c|c|c|c|}
\hline \multirow{2}{*}{$\begin{array}{l}\text { Basic characteristics } \\
\text { Age }\end{array}$} & \multirow[t]{2}{*}{ Numbers } & \multirow{2}{*}{$\begin{array}{l}\text { Serum EN01 assay values } \\
\text { (Mean } \pm \text { SD) }\end{array}$} & \multicolumn{2}{|c|}{ Significance } \\
\hline & & & & \\
\hline $\begin{array}{l}\text { Middle-aged and young } \\
\text { (<50 years) }\end{array}$ & 17 & $219.43 \pm 227.82$ & \multirow[t]{2}{*}{$t=1.786$} & \multirow[t]{2}{*}{$P=0.077$} \\
\hline Older ( $\geq 50$ years old) & 87 & $141.28 \pm 150.44$ & & \\
\hline \multicolumn{5}{|l|}{ Gender } \\
\hline Male & 88 & $168.59 \pm 176.94$ & \multirow[t]{2}{*}{$t=2.120$} & \multirow[t]{2}{*}{$P=0.036$} \\
\hline Female & 16 & $74.12 \pm 35.61$ & & \\
\hline \multicolumn{5}{|l|}{ AFP status } \\
\hline AFP negative & 21 & $122.39 \pm 113.06$ & \multirow[t]{2}{*}{$t=-0.974$} & \multirow[t]{2}{*}{$P=0.332$} \\
\hline AFP Positive & 83 & $162.07 \pm 177.44$ & & \\
\hline \multicolumn{5}{|l|}{ Tumor size } \\
\hline$<5 c$ & 27 & $90.55 \pm 68.09$ & \multirow[t]{2}{*}{$t=2.350$} & \multirow[t]{2}{*}{$P=0.021$} \\
\hline$\geq 5 c$ & 77 & $176.33 \pm 184.82$ & & \\
\hline \multicolumn{5}{|l|}{ Tumor metastatic or not } \\
\hline Yes & 64 & $185.74 \pm 191.52$ & \multirow[t]{2}{*}{$t=2.513$} & \multirow[t]{2}{*}{$P=0.14$} \\
\hline No & 40 & $103.38 \pm 99.46$ & & \\
\hline \multicolumn{5}{|l|}{ Recurrence of tumour } \\
\hline Yes & 32 & $234.77 \pm 261.27$ & \multirow[t]{2}{*}{$t=3.462$} & \multirow[t]{2}{*}{$P=0.001$} \\
\hline No & 72 & $118.19 \pm 79.29$ & & \\
\hline \multicolumn{5}{|l|}{ TNM grading } \\
\hline I & 33 & $86.76 \pm 69.68$ & $\begin{array}{l}\text { I vs || } \\
t=-2.285\end{array}$ & $P=0.795$ \\
\hline II & 14 & $179.83 \pm 210.95$ & $\begin{array}{l}\text { II vs III } \\
t=-0.262\end{array}$ & $P=0.557$ \\
\hline III & 39 & $196.08 \pm 195.35$ & $\begin{array}{l}\text { III vs IV } \\
t=0.561\end{array}$ & $P=0.027$ \\
\hline IV & 18 & $166.35 \pm 162.57$ & & \\
\hline
\end{tabular}




\section{Edmondson grading}

\begin{tabular}{|lllll|} 
Early (I-II) & 47 & $114.48 \pm 133.44$ & $t=-2.241$ & $P=0.027$ \\
\cline { 1 - 3 } & 57 & $186.70 \pm 184.70$ &
\end{tabular}

Legend囚AFP: alpha-fetoprotein; ENO1: a-enolasel; TNM: Tumor Node Metastasis $\$ The cut-off value for a positive AFP is $7 \mathrm{ng} / \mathrm{L}$.

\section{Analysis of the sensitivity of serum ENO1 in hepatocellular carcinoma and its correlation with AFP}

To explore the correlation between serum ENO1 levels and AFP levels by bivariate Spearman correlation analysis, the sensitivity and specificity of ENO1, AFP and their synergy in the diagnosis of liver cancer were analyzed by ROC curves (Figure 3). The results showed that serum ENO1 levels showed a significant positive correlation with AFP levels (Spearman's correlation coefficient $0.312, p=0.001^{\star \star}$ ), ROC curve analysis showed that ENO1 had a sensitivity of 0.673 and specificity of 0.831 (AUC=0.782, cutoff $=75.96$, $\mathrm{P}=0.0001$ ) in the diagnosis of $\mathrm{HCC}$ (distinguishing between healthy people and HCC patients) in this group, AFP had a sensitivity of 0.712 and a specificity of 0.729 ( $A U C=0.800$, cutoff $=27.02, P=0.0001)$ in the diagnosis of HCC in this group (distinguishing between healthy people and HCC patients). The sensitivity of ENO1 in concert with AFP in the diagnosis of HCC in this group was 0.740 and the specificity was 0.723 ( $\mathrm{AUC}=0.835, \mathrm{P}=0.0001$ ). The results suggest that ENO1 has a certain sensitivity and specificity in the diagnosis of hepatocellular carcinoma, and that ENO1 and AFP can perform synergistic diagnosis to improve the sensitivity of detection. (Figure 4)

\section{Diagnostic sensitivity and specificity of serum ENO1 levels in the clinical phenotype of hepatocellular carcinoma by ROC curve analysis}

The sensitivity and specificity of serum ENO1 levels in the clinical phenotype of hepatocellular carcinoma were analyzed by ROC curves, including tumor size, whether the tumor was metastatic or not, TNM staging, Edmondson grading, and whether the tumor was recurrent. The results showed that the AUCs of ENO1 in the diagnosis of tumor size, whether the tumor metastasized, TNM I stage, Edmondson grade and whether the tumor recurred in this group of HCC were $0.663\left(0.546-0.781, P=0.012^{\star}\right), 0.681(0.574-$ $\left.0.787, P=0.002^{\star *}\right), 0.553(0.421-0.686, P=0.386), 0.710(0.602-0.819, P=0.001 * \star)$ and $0.685(0.580-0.789$, $\left.\mathrm{P}=0.001^{\star \star}\right)$. The correlation results suggest that serum ENO1 levels can effectively discriminate tumor size, metastasis and stage grading in patients with hepatocellular carcinoma, further confirming its potential as a diagnostic marker for hepatocellular carcinoma, and further clarifying that ENO1 may play an important role in the development of hepatocellular carcinoma. (Figure 4)

\section{Distribution of serum ENO1 levels in the survival curve of patients with hepatocellular carcinoma}

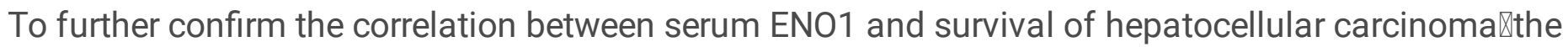
ENO1 assay values of 104 patients with hepatocellular carcinoma were divided into two groups according to the ROC curve cutoff values, $0<$ EN01 $\leq 75.96$ and EN01>75.96 respectively, and the 
correlation between serum ENO1 levels and patients' biological curves was analyzed by survival curves. The results showed that serum ENO1 levels were significantly correlated with the survival of patients with hepatocellular carcinoma, Within 40 months, patients with high ENO1 levels survived significantly less than those with low ENO1 levels $(p<0.05)$ (Figure 5). The correlation results suggest that ENO1 levels can provide a guide and basis for patient survival after surgery.

\section{Discussion}

The incidence of hepatocellular carcinoma (HCC) is extremely high in our country and is a serious health risk to patients. Early diagnosis of hepatocellular carcinoma has a tremendous contribution to improving the treatment outcome of patients with hepatocellular carcinoma. At present, the most commonly used serum marker in hepatocellular carcinoma clinics is AFP, which is a histone protein secreted through primitive embryonic tissue, and its ability to be specifically expressed in the serum of hepatocellular carcinoma patients, thus playing a role in establishing the diagnosis or differential diagnosis. However, some studies have found that there are limitations to the application of AFP in the diagnosis of hepatocellular carcinoma, because serum AFP also abnormally upregulated in patients with cirrhosis, viral hepatitis, reproductive tumors, or metabolic syndrome ${ }^{[20]}$. In addition, there are false negatives for AFP in the diagnosis of hepatocellular carcinoma, and all of these factors limit the practicality of AFP in the clinical adjunct diagnosis of hepatocellular carcinoma to some extent ${ }^{[21]}$. Fortunately, other hepatocellular carcinoma diagnostic markers can work synergistically with AFP to improve diagnostic efficiency. For example, abnormal prothrombin (Des-gamma-carboxyprothrombin, DCP) is undetectable in the serum of healthy populations, but is abnormally highly expressed in the serum of patients with hepatocellular carcinoma, which has a sensitivity of $56 \%$ for the diagnosis of hepatocellular carcinoma alone, shows greater specificity in the characterization of hepatocellular carcinoma invasion, and is a serum marker for differentiating benign/malignant hepatocellular carcinoma ${ }^{[22]}$. Golgi Protein 73 (GP73), also upregulated in expression during hepatocarcinogenesis and lowly expressed in hepatocytes of healthy population, has a sensitivity of about 76\% for diagnosis alone, and GP73 is able to synergize with AFP to improve the sensitivity and specificity of hepatocellular carcinoma diagnosis ${ }^{[23]}$. The sensitivity of interleukin IL- 6 alone in diagnosing early-stage hepatocellular carcinoma was only $46 \%$, but its serum levels showed a significant positive correlation with AFP levels and could complement the AFP diagnosis to some extent ${ }^{[24]}$. However, the tissue specificity of IL-6 is extremely poor, and its expression can be upregulated in a variety of diseases and multiple organs, thus limiting its diagnostic value, but the idea of finding positive AFP-related markers deserves further in-depth study. Therefore, exploring new HCC serological markers can effectively improve the detection rate and detection sensitivity of early hepatocellular carcinoma.

A-enolase (a-enolase, EN01) is one of the key enzymes of the glycolytic pathway, which acts as a glycolytic rate-limiting enzyme in the cytoplasm to regulate the metabolism of tumor cells and thus affect their proliferation. It functions as a fibrinogen receptor on the cell surface, accelerating the degradation of the extracellular matrix and thus affecting tumor cell invasion and migration, and in the nucleus, it acts 
as a transcriptional repressor through encoded proteins and thus affects tumorigenesis and progression. Therefore, ENO1 is closely related to tumorigenesis and development and is a potential excellent target for studying tumor-related mechanisms. Reports have confirmed that ENO1 is aberrantly expressed in tumor tissues of non-small cell lung cancer ${ }^{[25]}$, liver cancer ${ }^{[20]}$, colorectal cancer ${ }^{[26]}$, and endometrial cancer ${ }^{[26]}$, and could be a potential diagnostic marker for these tumors. Through preliminary studies and bioinformatic analysis of ENO1-related data in existing databases, we found that the relative expression of both mRNA and protein of ENO1 was significantly up-regulated in hepatocellular carcinoma tissues, which, together with the literature, further confirmed its ability to serve as a potential diagnostic marker for hepatocellular carcinoma ${ }^{[27]}$.

In this study, 28 serum samples of chronic hepatitis B (CHB), 31 serum samples of cirrhosis (LC), 56 serum samples of hepatocellular carcinoma (HCC) and 26 serum samples of healthy population were collected. The levels of ENO1 in the serum of different populations were detected, and the results showed that the expression of ENO1 in HCC was sequentially higher than that in the CHB group, the LC group, and the healthy population. This suggests that serum ENO1 levels are significantly associated with liver disease and its specific high expression in the HCC group indicates its potential as a marker for hepatocellular carcinoma diagnosis. The related findings are consistent with the existing reports ${ }^{[15-16]}$. In addition, the expression of ENO1 in the serum of HCC patients was significantly higher than its expression in $\mathrm{CHB}$ patients and LC patients, indicating that it possesses tissue specificity along with the specificity of HCC disease, avoiding the interference of other liver diseases with the diagnostic results of ENO1, a property that makes ENO1 more potential and valuable than IL-6 in hepatocellular carcinoma.

The correlation between the distribution of serum ENO1 levels and hepatitis B virus levels in liver disease in the CHB, LC, and HCC groups was investigated by curvilinear regression analysis. The results showed that hepatitis B virus DNA levels were significantly and positively correlated with ENO1 levels in CHB patients, whereas no statistically significant correlation was found between hepatitis $B$ virus DNA contents and ENO1 levels in LC and HCC patients. The correlation results suggest that the elevated ENO1 levels may be associated with hepatitis B virus infection. Unfortunately, at the time of patient inclusion and exclusion, we hepatitis B-induced hepatocellular carcinoma and cirrhosis were set as exclusion criteria, which limited the results of the correlation between hepatitis B virus DNA levels and ENO1 levels in LC and HCC patients. In contrast, related reports have shown that ENO1 is able to act as an autoantigen to stimulate the body's immune system and induce local inflammation [28]. The expression of ENO1 can be detected in the serum of patients with various autoimmune diseases, such as autoimmune hepatitis, systemic lupus erythematosus, and rheumatoid arthritis ${ }^{[29]}$. Therefore, it is reasonable to suspect that ENO1 plays a role in the host immune process and lesions caused by hepatitis B virus infection of the host, and the related mechanisms need to be studied in depth. The diagnostic efficiency of hepatitis B virus on ENO1 in HCC can only be truly ruled out if ENO1 can be detected in patients who do not exclude hepatitis $B$ virus infection leading to hepatocellular carcinoma and confirm that the high expression of ENO1 in the serum of HCC patients is not affected by hepatitis B virus levels. 
The results of the distribution of serum ENO1 levels in different clinical phenotypes of hepatocellular carcinoma showed that the differences in the distribution of serum ENO1 levels in clinical data subgroups AFP status, TNM stage (II vs III, III vs IV) were not statistically significant ( $p>0.05)$. There were statistically significant differences in the distribution of clinical data subgroups gender, tumor size, whether the tumor metastasized, TNM stage (I vs II), and Edmondson's grading ( $<<0.05)$, and highly significant differences in the distribution of clinical data subgroups whether the tumor recurred $(p<0.01)$. A study by TAKASHIMA ${ }^{[30]}$ confirmed that high expression of ENO1 was closely associated with tumor size and tumor recurrence in hepatocellular carcinoma, and our experiments verified this conclusion from ENO1 serum levels. In addition, previous studies have shown that high expression of ENO1 promotes tumor cell proliferation and invasion, accelerates tumor metastasis, and inhibits tumor cell apoptosis ${ }^{[7-}$ 11]. This suggests that the findings of ENO1 at the cellular level are consistent with those at the serum level. The related findings further suggest that serum ENO1 can be used as a marker for early hepatocellular carcinoma diagnosis and hepatocellular carcinoma recurrence diagnosis.

On the basis that serum ENO1 can be used as a clinical diagnostic marker for hepatocellular carcinoma. We explored the correlation between serum ENO1 levels and AFP levels by bivariate Spearman correlation analysis, and analyzed the sensitivity and specificity of ENO1, AFP and their synergy in the diagnosis of hepatocellular carcinoma by ROC curves. The results showed that serum ENO1 levels showed a significant positive correlation with AFP levels; ROC curve analysis showed that the diagnostic sensitivity of ENO1 in this group of HCC patients was $67.3 \%$ (specificity was $83.1 \%$ ). In comparison, the sensitivity and specificity of AFP were $71.2 \%$ and $72.9 \%$, respectively. If ENO1 and AFP were used for co-detection, the sensitivity was increased to $74 \%$. The correlation results suggest that ENO1 can be used not only as a diagnostic test for hepatocellular carcinoma in isolation, but also in combination with AFP. Its advantage was higher than molecular markers of hepatocellular carcinoma such as abnormal prothrombin (DCP) and interleukin IL-6, and slightly weaker than phosphatidylinositol proteoglycan 3 (GPC3) and Golgi glycoprotein 73 (GP73).

Subsequently, we further explored the diagnostic sensitivity of serum ENO1 levels in the clinical phenotype of hepatocellular carcinoma by ROC curves, and the results showed that the AUCs of ENO1 in the diagnosis of tumor size, whether the tumor was metastatic, Edmondson grade, and whether the tumor was recurrent in this group of HCC were $0.663,0.681,0.710$, and 0.685 , respectively $(0.580-0.789$, $\left.\mathrm{P}=0.001^{\star \star}\right)$. This suggests that serum ENO1 has potential not only for diagnosing the occurrence of hepatocellular carcinoma, but also for providing some auxiliary identification of tumor size, metastasis, and grading and staging. To further confirm the correlation between serum ENO1 and survival of hepatocellular carcinoma, the ENO1 detection values of 104 hepatocellular carcinoma patients were divided into two groups according to ROC curve cutoff values of $0<$ ENO1 $\leq 75.96$ and ENO1> 75.96, and the correlation between serum ENO1 levels and patients' biological curves was analyzed by survival curves. The results showed that serum ENO1 levels were significantly correlated with postoperative survival in patients with hepatocellular carcinoma, and patients with high ENO1 levels survived significantly less than those with low ENO1 levels. This suggests that ENO1 levels can provide guidance 
and basis for postoperative survival of patients. This is consistent with the findings of related reports ${ }^{\text {[31] }}$. However, we did not find other relevant reports that studied ENO1 from the serum marker level, so it was not possible to make comparisons and corrections to the available data. However, the relevant data have been able to clarify that ENO1 is a new serological marker for the diagnosis of hepatocellular carcinoma. Further data cohorts and patient sub-groups need to be expanded for more in-depth studies and analyses.

Notably, it can be speculated from our data that ENO1 levels are significantly associated with migration, invasion, and progression of hepatocellular carcinoma. The mechanism of ENO1 in hepatocarcinogenesis and progression also possesses research value. Interference with ENO1 was reported to be effective in inhibiting cell proliferation, migration and invasion of human hepatoma cells HepG2 at the cellular level ${ }^{[16]}$. Some reports found that ENO1 may regulate biological functions such as proliferation and migration of hepatocellular carcinoma cells through Notch signaling pathway. There are also reports showing that ENO1 in tumor cells can be able to be activated by glycolytic enzymes, and thus expression is upregulated to function with Warburg effect, enhancing glucose metabolism in tumor cells, and thus accelerating tumor cell proliferation ${ }^{[31]}$. However, the specific mechanism of these speculations has not been elucidated, and further in-depth studies are needed.

In summary, we concluded that ENO1 plays an important role in the development, metastasis, and healing of hepatocellular carcinoma. ENO1 is a new diagnostic serum marker for hepatocellular carcinoma, which plays an important auxiliary diagnostic function in the confirmation, staging and grading, evaluation of metastasis, and healing and recurrence stages of hepatocellular carcinoma. The evaluation of its sensitivity and specificity requires statistical analysis of a larger cohort sample, and the associated mechanisms deserve in-depth study, which is the direction of our further research.

\section{Conclusion}

Serum ENO1 levels were significantly correlated with the occurrence, progression, metastasis, staging and grading of hepatocellular carcinoma, and postoperative survival of patients. ENO1 may serve as a candidate diagnostic marker for early hepatocellular carcinoma diagnosis and evaluation of hepatocellular carcinoma progression, and is able to perform an auxiliary diagnostic function in combination with AFP.

\section{Abbreviations}

ENO1: alpha-enolase; AFP: alpha fetoprotein; HCC: Hepatocellular carcinoma; HBV: Hepatitis B virus; HCV: hepatitis C virus; CHB: chronic hepatitis B; LC: liver cirrhosis; TNM: Tumor Node Metastasis;

\section{Declarations}

\section{Ethics approval and consent to participate}


The study protocols were approved by the General Hospital of Ningxia Medical University Ethics Committee (Yinchuan, China) and all methods were performed in accordance with the principles expressed in the Declaration of Helsinki. All patients provided written informed consent to participate.

\section{Consent for publication}

Not applicable.

\section{Availability of data and material}

All data generated or analysed during this study are included in this published article.

\section{Competing interests}

The authors declare that they have no competing interests

\section{Funding}

This work was supported by grants from the Key Projects of Ningxia Natural Science Foundation (2018AAC02014) the National Natural Science Foundation of China (Nos. 81760363) and Research Program of Ningxia Medical University (XM2018113)

\section{Authors' contributions}

Xia Luo: designed project, participated in all experiment, and wrote paper.

Xiangchun Ding: Guided the experiment and technology

Lina Ma: took part in designing project and revised article.

Shuaiwei Liu: Analyzed the data of the experiment

Long Hai: Did molecular experiment and revised grammar.

\section{Acknowledgements}

Not applicable.

\section{References}

1. International Agency for Research on Cancer. World Health Organization, France. 2021. https://gco.iarc.fr/today/home. Accessed 18 Feb, 2021

2. Wu SS., Shan QY, Xie WX, et al. Outcomes after hepatectomy of patients with positive HBcAb Non-B Non-C hepatocellular carcinoma compared to overt hepatitis B virus hepatocellular carcinoma. Clin Transl Oncol, 2020, 22(3): 401-410. 
3. Zucman-Rossi J, Villanueva A, Nault J C, et al. Genetic landscape and biomarkers of hepatocellular carcinoma. Gastroenterology, 2015, 149(5): 1226-1239. e4.

4. Chen W, Sun K, Zheng R, et al. Cancer incidence and mortality in China, 2014. Chinese J Cancer Res, 2018, 30(1): 1.

5. Zhang ZW, Zhen XP, Code of Practice for the Treatment of Primary Liver Cancer, 2017, J Clin Laser Med Sur, 2018, 26(1): 5-8.

6. Ejeskär K, Krona C, Carén $\mathrm{H}$, et al. Introduction of in vitro transcribed ENO1 mRNA into neuroblastoma cells induces cell death. BMC Cancer, 2005, 5: 161.

7. Jiang K, Dong C, Yin Z, et al. Exosome-derived ENO1 regulates integrin a6ß4 expression and promotes hepatocellular carcinoma growth and metastasis. Cell Death Dis, 2020, 11(11): 1-20.

8. Huang YF, Progress in the functional study of enolase in model organisms. Metallurgy and Materials, 2019, 39(04):15-17.

9. Almaguel FA, Sanchez TW, Ortiz-Hernandez GL, et al. Alpha-Enolase: Emerging Tumor-Associated Antigen, Cancer Biomarker, and Oncotherapeutic Target. Front Genet, 2020, 11: 1835.

10. Dai J, Zhou Q, Chen J, et al. Alpha- enolase regulates the malignant phenotype of pulmonary artery smooth muscle cells via the AMPK-Akt pathway. Nat Commun, 2018, 9(1): 3850.

11. Zhang L, Lu T, Yang Y, et al. a-enolase is highly expressed in liver cancer and promotes cancer cell invasion and metastasis. Oncol Lett, 2020, 20(5): 1-1.

12. Pan D, Chen J, Feng $C$, et al. Preferential localization of MUC1 glycoprotein in exosomes secreted by non- small cell lung carcinoma cells. Int J Mol Sci, 2019, 20(2).

13. Shen $\mathrm{AH}, \mathrm{Li} \mathrm{YH}$, Su JC, et al. Expression and significance of FBXW7 and ENO1 in high- and low-grade plasmacytoid adenocarcinoma of the ovary. Chinese Comprehensive Clinical, 2018, 34(6): 496-502.

14. Mittal L, Aryal UK, Camarillo IG, et al. Quantitative proteomic analysis of enhanced cellular effects of electrochemotherapy with cisplatin in triple- negative breast cancer cells. Sci Rep, 2019, 9(1): 13916.

15. Zhu W, Li H, Yu Y, et al. Enolase-1 serves as a biomarker of diagnosis and prognosis in hepatocellular carcinoma patients. Cancer Manag Res, 2018, 10: 5735-5745.

16. Yan TT, Ma LN, Luo X, et al. The role of alpha-enolase in hepatocellular liver cancer and its mechanism. Chinese Journal of Liver Diseases, 2017, 25(6): 429-434.

17. Qi R, Wang J, Jiang Y, et al. Snai1-induced partial epithelial-mesenchymal transition orchestrates p53-p21-mediated G2/M arrest in the progression of renal fibrosis via NF-KB-mediated inflammation[J]. Cell Death Dis, 2021, 12(1): 1-15.

18. Fromer MW, Aloia TA, Gaughan JP, et al. The utility of the MELD score in predicting mortality following liver resection for metastasis. European Journal of Surgical Oncology (EJSO), 2016, 42(10): 1568-1575.

19. Gershenwald JE, Scolyer RA, Thompson JF, et al. Melanoma staging: Evidence-based changes in the American Joint Committee on Cancer eighth edition cancer staging manual. CA Cancer J Clin. 2017 Oct 13 . 
20. Bai DS, Zhang C, Chen P, et al. The prognostic correlation of AFP level at diagnosis with pathological grade, progression, and survival of patients with hepatocellular carcinoma. Sci Rep, 2017, 7(1): 1-9.

21. Bertino G, Neri S, Bruno CM, et al. Diagnostic and prognostic valueof alpha-fetoprotein, des-y-carboxy prothrombin and squamous cell carcinoma antigen immunoglobulin $\mathrm{M}$ complexes in hepatocellular carcinoma. Minerva Med, 2011, 102(5): 363-371.

22. Lok AS, Sterling RK, Everhart JE, et al. Des-y-carboxy Prothrombin and a-fetoprotein as Biomarkers for the Early Detection of Hepatocellular Carcinoma. Gastroenterology,2010,138(2): 493-502.

23. Xu WJ, Guo BL, Han YG, et al. Retarcted Article: Diagnostic Value of Alpha-fetoprotein-L3 and Golgi Protein 73 in Hepatocellular Carcinomas with Low AFP Levels. Tumor Biol,2014,35(12): 1206912074.

24. Tang $L$, Zhang WH, Zhu X. The significance of IL-6 levels in the early diagnosis of primary hepatocellular carcinoma. Public Health and Preventive Medicine,2012,23(1): 40-43.

25. Fu Q F, Liu Y, Fan Y, et al. Alpha-enolase promotes cell glycolysis, growth, migration, and invasion in non-small cell lung cancer through FAK-mediated PI3K/AKT pathway. J Hematol Oncol, 2015, 8(1): $1-13$.

26. Hong J, Guo F, Lu S Y, et al. F. nucleatum targets IncRNA ENO1-IT1 to promote glycolysis and oncogenesis in colorectal cancer. Gut, 2020.

27. Gong W, Ekmu B, Wang X, et al. AGR2-induced glucose metabolism facilitated the progression of endometrial carcinoma via enhancing the MUC1/HIF-1a pathway. Hum Cell 2020, 33(3): 790-800.

28. Tan HT, Low J, Lim SG, et al. Serum autoantibodies as biomarkers for early cancer detection. Febs Journal, 2009, 276(23):6880-6904.

29. Ji H, Wang J, Guo J, et al. Progress in the biological function of alpha-enolas. Anim Nutr 2016, 2(1): 12-17.

30. Takashima M, Kuramitsu Y, Yokoyama Y, et al. Overexpression of alpha enolase in hepatitis $C$ virusrelated hepatocellular carcinoma: Association with tumor progression as determined by proteomic analysis. Proteomics, 2005, 5(6): 1686-1692.

31. Zhu W, Li H, Yu Y, et al. Enolase-1 serves as a biomarker of diagnosis and prognosis in hepatocellular carcinoma patients. Cancer Manag Res, 2018, 10: 5735.

\section{Figures}



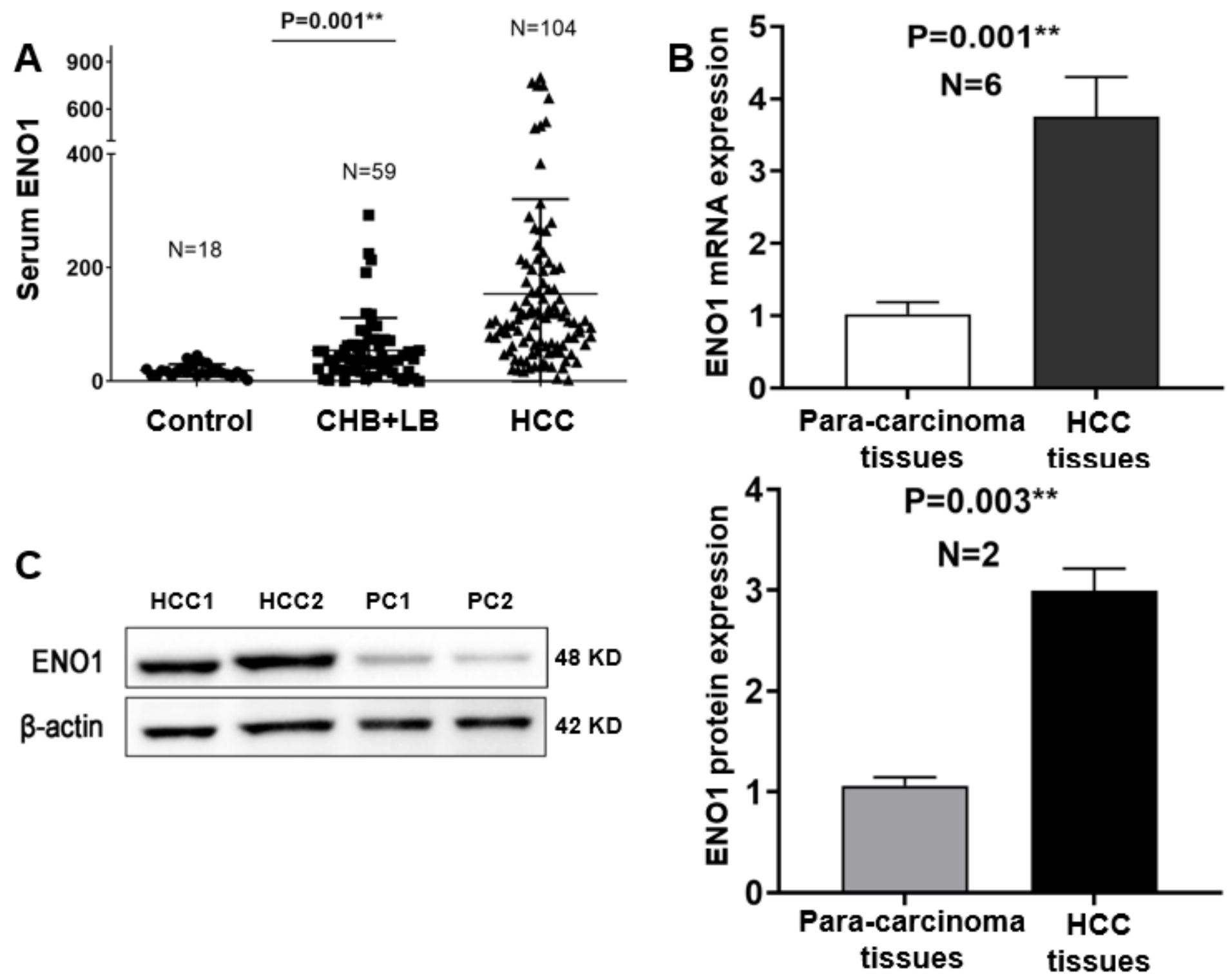

Figure 1

Differential expression of ENO1 in normal population/non-hepatocellular carcinoma liver disease patients/hepatocellular carcinoma patients and in hepatocellular carcinoma/paraneoplastic tissues 

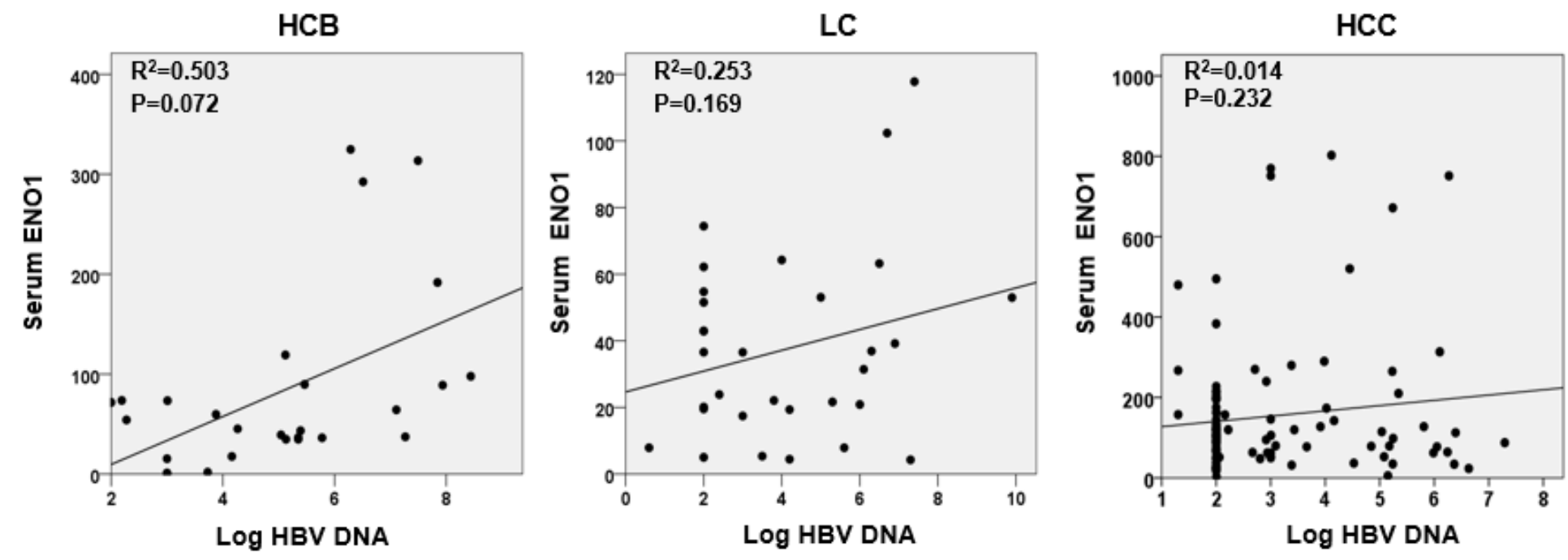

Figure 2

Correlation between serum ENO1 levels and hepatitis B virus DNA levels

A

\begin{tabular}{|c|c|c|c|}
\hline \multicolumn{2}{|c|}{$\begin{array}{c}\text { Spearman } \\
\text { Rho }\end{array}$} & $\begin{array}{l}\text { ENO1 } \\
\text { (mg/L) }\end{array}$ & $\begin{array}{c}\text { AFP } \\
(\mathrm{ng} / \mathrm{mL})\end{array}$ \\
\hline \multirow{3}{*}{$\begin{array}{l}\text { ENO1 } \\
\text { (mg/L) }\end{array}$} & $\begin{array}{c}\text { correlation } \\
\text { coefficient }\end{array}$ & 1 & $0.312^{* *}$ \\
\hline & P-value & & $0.001^{* *}$ \\
\hline & $\mathrm{N}$ & 104 & 104 \\
\hline \multirow{3}{*}{$\begin{array}{c}\text { AFP } \\
(\mathrm{ng} / \mathrm{mL})\end{array}$} & $\begin{array}{l}\text { correlation } \\
\text { coefficient }\end{array}$ & $0.312^{\star *}$ & \\
\hline & P-value & $0.001^{\star \star}$ & \\
\hline & $\mathrm{N}$ & 104 & 104 \\
\hline
\end{tabular}

${ }^{\star} . \mathrm{P}<0.05$, Significant correlation;

**. $\mathrm{P}<0.01$, Highly significant correlation.

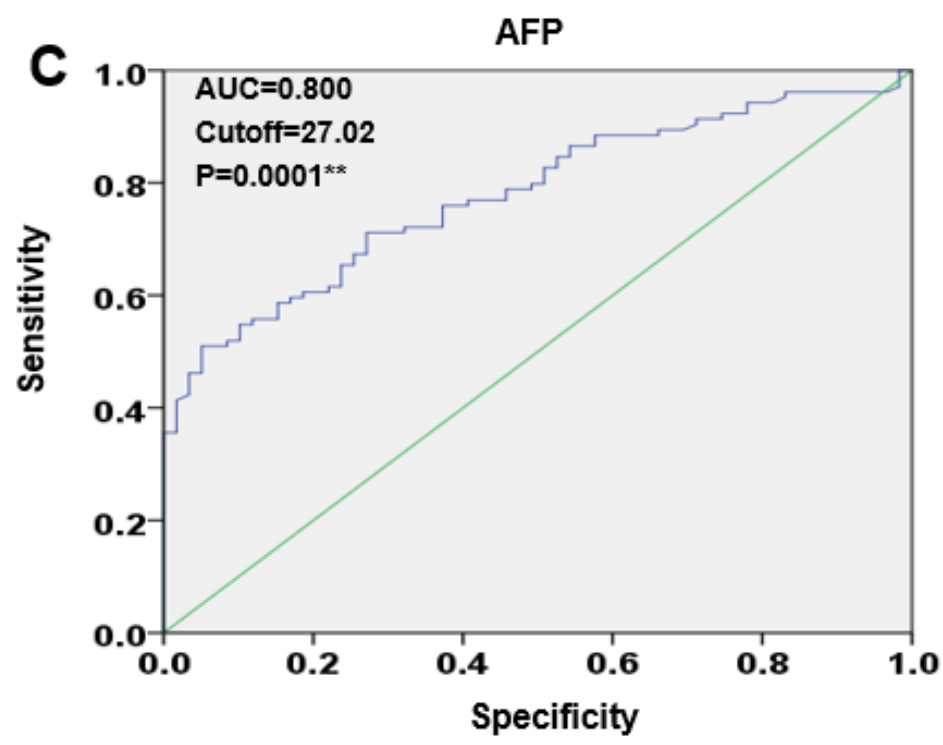

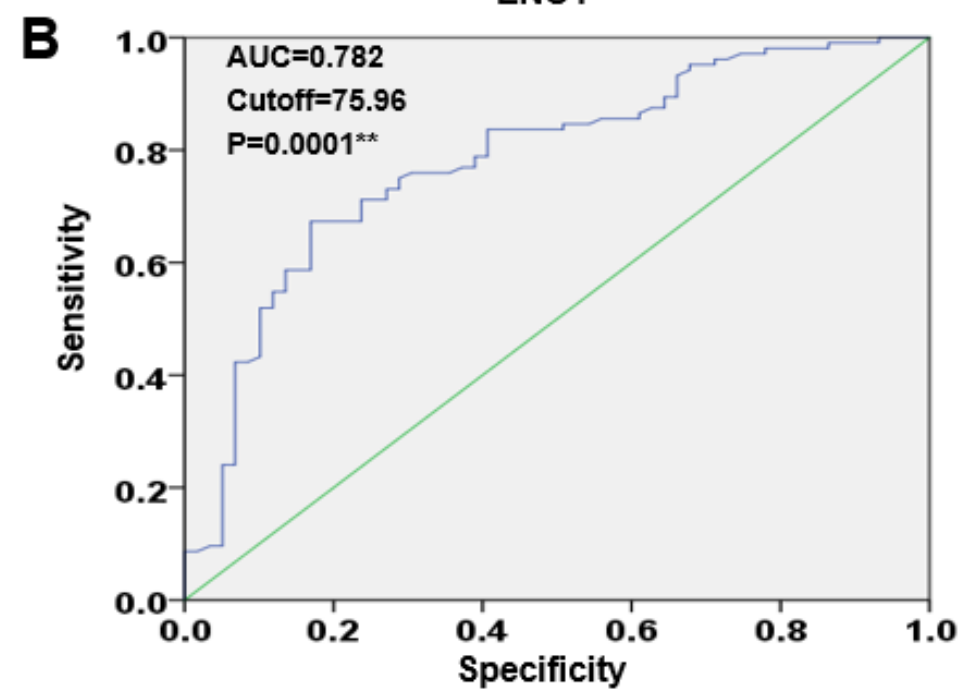

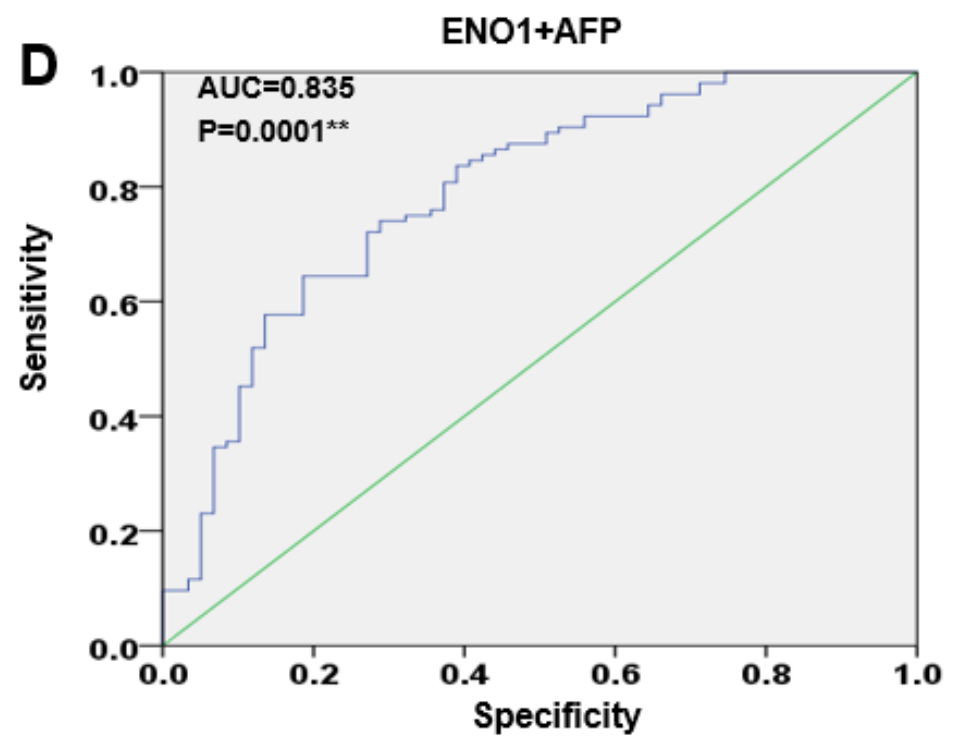


Figure 3

Correlation between serum ENO1 and AFP. A. Bivariate Spearman correlation analysis. B. ROC curve for ENO1 diagnosing hepatocarcinogenesis. C. ROC curves for AFP diagnosis of hepatocarcinogenesis. D. ROC curve of ENO1 in collaboration with AFP for the diagnosis of hepatocarcinogenesis
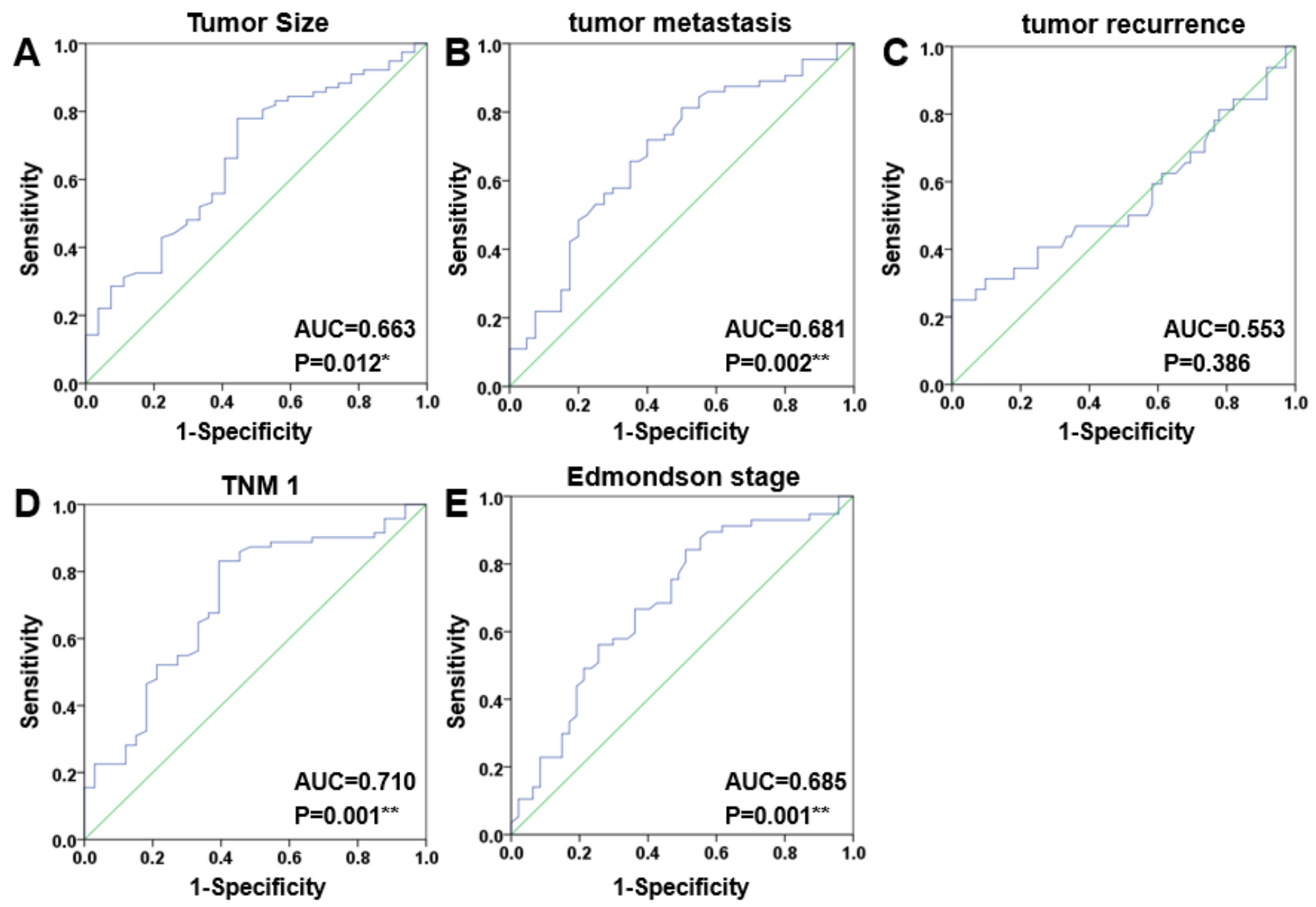

Figure 4

Diagnostic value of serum ENO1 levels for clinical phenotyping of tumors by ROC curve analysis AUCarea under the curve. A. ENO1 level to diagnose tumor maximum diameter $>5 \mathrm{~cm}$. B. ENO1 level to diagnose tumor metastasis, and C. ENO1 level to diagnose tumor recurrence. D. ENO1 level to diagnose TNM I staging. E. ENO1 level diagnoses early stage of tumor Edmondson classification (TNM I/I I stage), ${ }^{*} \mathrm{P}<0.05 ; * \star \mathrm{P}<0.01$ 


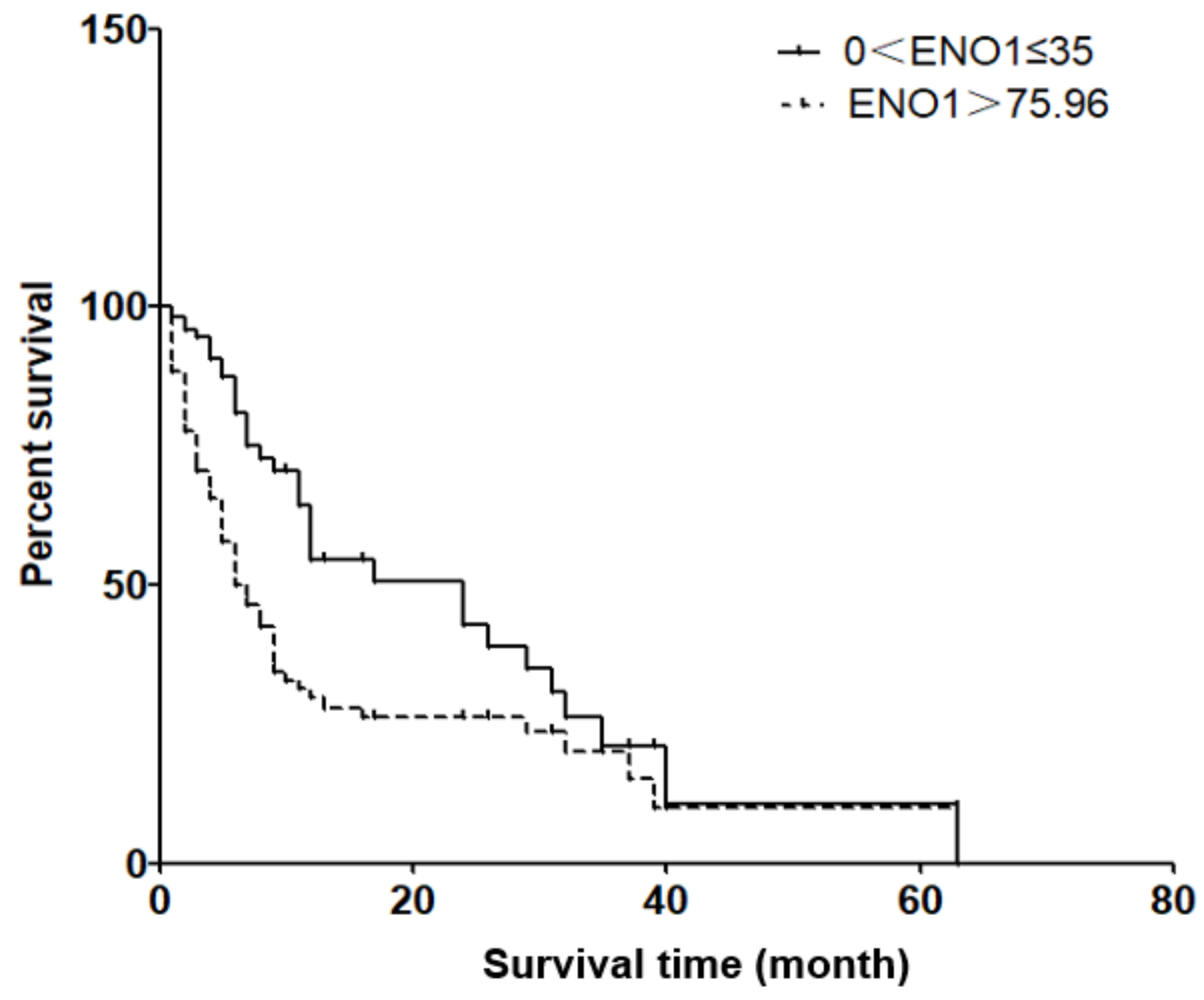

Figure 5

Distribution of serum ENO1 levels in the survival curve of patients with hepatocellular carcinoma 